\title{
A new method for deducing the effective collision frequency profile in the D-region
}

\author{
M. Beharrell ${ }^{1}$ and F. Honary ${ }^{1}$ \\ Received 17 July 2007; revised 1 October 2007; accepted 14 January 2008; published 3 May 2008. \\ [1] A method based on linear regularization is presented for the deduction of the effective \\ collision frequency profile in the D-region using an incoherent scatter radar and a \\ colocated riometer. The deduced collision frequency above EISCAT, Tromsø, for the \\ period 09 November 2004 to 13 November 2004 matches very well with that calculated \\ using neutral densities and temperatures from the NRLMSISe-00 atmospheric model \\ between $68 \mathrm{~km}$ and $95 \mathrm{~km}$ during active $(a p>50)$ and quiet $(a p<50)$ conditions. We also \\ confirm the validity of using the Appleton-Hartree equation with effective collision \\ frequencies when calculating the ionospheric absorption at high frequencies.
}

Citation: Beharrell, M., and F. Honary (2008), A new method for deducing the effective collision frequency profile in the D-region, J. Geophys. Res., 113, A05303, doi:10.1029/2007JA012650.

\section{Introduction}

[2] The ionospheric absorption of cosmic radio noise, measured by a riometer, is a function of electron-neutral momentum-transfer collision frequency and electron density, integrated over the path length [Davies, 1966]. The aim of this work is to apply linear regularization to a large (several thousand) number of absorption measurements with corresponding profiles of electron density in order to infer a collision frequency profile of the ionospheric D-region, which is responsible for the majority of absorption at high frequencies.

[3] Additionally, the method herein can reveal whether the electron density data at any given altitude and time are contributing more or less than expected to the groundmeasured absorption.

[4] Previously, Miyazaki [1975] obtained a collision frequency profile by combining eight rocket-measured electron density profiles of the Antarctic D-region, with corresponding ground-based cosmic noise absorption measurements. By assuming a log linear altitude dependence, the least squares method was employed to give a collision frequency profile of the form $\nu=\nu_{0} \exp (-h / H)$, where $h$ is the height above ground level and $H$ is the scale height.

[5] Serafimova and Serafimov [1991] proposed a method utilizing two ground-absorption measurements, together with ionosonde measurements, to arrive at an approximate exponential collision frequency profile of the ionosphere. Gokov et al. [1991] described a technique to simultaneously estimate electron density and electron-neutral collision frequency profiles, based on measurements of differential absorption and phase differences of the $\mathrm{O}$ and $\mathrm{X}$ components of partial reflection (PR) signals. This was used at Khar'kov State University to measure electron density and collision frequency profiles to an accuracy of better than $30 \%$.

\footnotetext{
${ }^{1}$ Department of Communication Systems, University of Lancaster, United Kingdom.

Copyright 2008 by the American Geophysical Union. 0148-0227/08/2007JA012650\$09.00
}

[6] Similarly, Vuthaluru et al. [2002] calculated collision frequency profiles in the low latitude D-region by partial reflection differential absorption and differential phase, using the large MF radar at Buckland, Australia. Though relying on atmospheric models for the extrapolation of results from lower altitudes, a good agreement with CIRA-86 calculated collision frequencies was reported below $80 \mathrm{~km}$.

[7] Such investigations are critical in assessing and improving models of the upper atmosphere, in which systematic errors can be a significant problem, often caused by the interpolation and extrapolation of empirical data that does not cover a wide enough range of locations, times, or conditions. Drob and Picone [2000] report that two previous models, MSISe-90 [Hedin, 1991] and CIRA-86 [Flemming et al., 1988], are both thought to underestimate and overestimate the mesopause region temperatures by around $15 \mathrm{~K}$, at midlatitudes and high-latitudes respectively. They explore the shortcomings of such atmospheric models in an area where their performance would be of great importance, that of the potential infrasonic monitoring of a Comprehensive Nuclear-Test-Ban Treaty (CTBT). As a function of neutral density and temperature, the electron-neutral effective momentum-transfer collision frequency can, to some extent, act as a proxy for these parameters in a test of the accuracy of an atmospheric model.

[8] The primary result of this study is in the form of timeaveraged correction factors for the effective collision frequency profile calculated with the NRLMSISe-00 neutral atmosphere model, for active $(a p>50)$ and quiet $(a p<50)$ conditions over the period 2004-11-09 to 2004-11-13. The method would be equally effective if results were to be split between, for example, heater on and heater off periods of a colocated ionospheric heating facility.

\section{Instruments}

[9] The European Incoherent Scatter (EISCAT) VHF radar [Baron, 1986] measures the basic parameters of the 


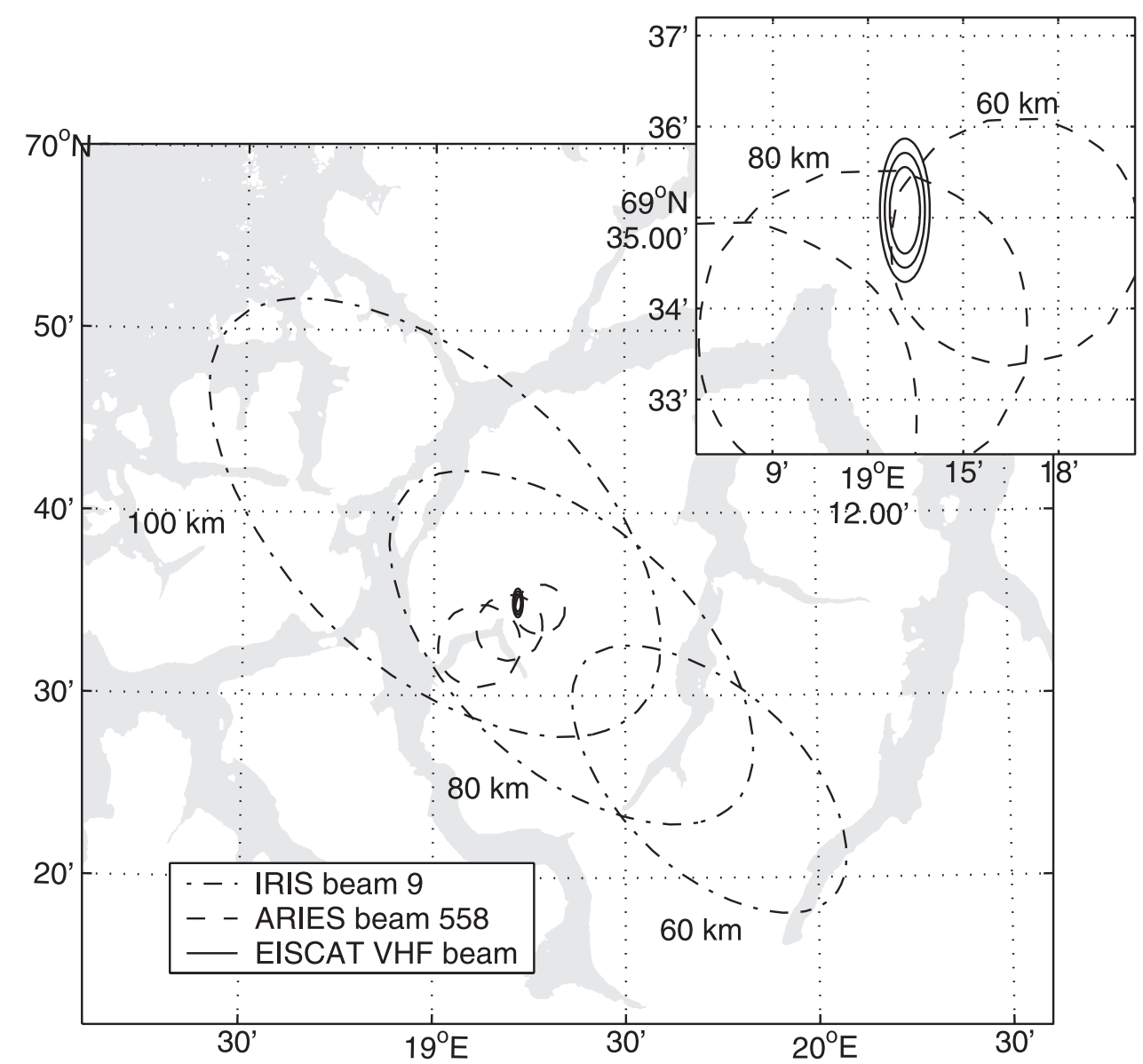

Figure 1. Map showing the location of beams 9 of IRIS and 558 of ARIES with the EISCAT VHF beam, at altitudes of 60,80 , and $100 \mathrm{~km}$. Inset: Close-up of the relatively narrow VHF radar beam.

ionosphere from an altitude of $60 \mathrm{~km}$ to $1600 \mathrm{~km}$. It is located near Tromsø $\left(69^{\circ} 35^{\prime} \mathrm{N}, 19^{\circ} 14^{\prime} \mathrm{E}\right)$. Between $2004-$ 11-09 and 2004-11-13, the VHF radar was operating under the arc_dlayer-v program, which provided electron density measurements for the altitude range $60-140 \mathrm{~km}$.

[10] The Imaging Riometer for Ionospheric Studies (IRIS) [Browne et al., 1995], comprises a $7 \times 7$ array of 49 riometer beams, acheiving a maximum angular resolution of $13^{\circ}$ at zenith. It measures the ionospheric absorption of X-mode cosmic noise above Kilpisjarvi $\left(69^{\circ} 03^{\prime} \mathrm{N}\right.$, $20^{\circ} 47^{\prime} \mathrm{E}$ ) at $38.2 \mathrm{MHz}$, sampling all beams once per second. IRIS is located approximately $83 \mathrm{~km}$ from the EISCAT VHF radar with beam 9 passing directly over EISCAT, see Figure 1. Also shown is the projection of beam 558 of ARIES [Grill et al., 2003]. ARIES is a new type of riometer with a much higher angular resolution than IRIS (max. $4.75^{\circ}$ at zenith). It receives at $38.2 \mathrm{MHz}$, and is located just $12.7 \mathrm{~km}$ from EISCAT.

\section{Theory}

[11] The Appleton-Hartree equation for the absorption coefficient, $\kappa$, of monoenergetic electrons (i.e., electrons sharing the same collision frequency) is:

$$
\kappa=\frac{e^{2}}{2 m_{e} c \epsilon_{0}} \cdot \frac{1}{\mu} \cdot \frac{N_{e} \nu_{m}}{\nu_{m}^{2}+\left(\omega \pm \omega_{H} \cos \theta\right)^{2}} \quad \mathrm{~Np} / \mathrm{m}
$$

[Davies, 1966] where $\nu_{m}$ is the energy-dependent electronneutral momentum-transfer collision frequency, $\mu$ is the real part of the refractive index, $\omega$ is the incident angular wave frequency, $\omega_{H}$ is the electron gyrofrequency, $\theta$ is the angle between the external magnetic field and the direction of phase-propagation of the incident wave, and $N_{e}$ is the electron density. The \pm applies to absorption of the O-mode and $\mathrm{X}$-mode respectively.

[12] This is identical to the approximation by Sen and Wyller [1960] when their primary assumption is applied, namely that the collision frequency is proportional to the electron energy $\left(\nu_{m} \propto v^{2}\right)$ and the function $\kappa$ is integrated over the electron energy distribution using the ChapmanEnskog method [Chapman and Cowling, 1970; Itikawa, 1971]. The Appleton-Hartree method has the advantage that a collision frequency function with any chosen dependence on electron velocity may be used.

[13] In the $\mathrm{D}$ and lower $\mathrm{E}$ regions absorption is largely non-deviative, so $\mu \approx 1$ may be assumed. Also, for the receiving frequency of IRIS, $\nu_{m}^{2}$ above $\sim 60 \mathrm{~km}$ is small compared to $\omega^{2}$, allowing integration over the electron distribution to be performed to the collision frequency alone - rather than the entire function - resulting in an effective collision frequency:

$$
\nu_{\text {eff }}=\sum_{\text {neutral species }}\left\langle\nu_{e, \text { neutral species }}\right\rangle .
$$


The angled brackets denote an average over the electron energy distribution, which is assumed to be Maxwellian. Converting from Nepers to decibels and integrating over the path length, (1) becomes:

$$
\text { Absorption }=4.6 \times 10^{-5} \int \frac{N_{\mathrm{e}} \nu_{\mathrm{eff}}}{\nu_{\mathrm{eff}}^{2}+\left(\omega \pm \omega_{\mathrm{H}} \cos \theta\right)^{2}} \mathrm{dl} \mathrm{dB} .
$$

[14] Kinetic calculations for the general refractive index [Garrett, 1985] are employed to investigate the validity of the simplifications made in reaching (3).

[15] The maximum electron density encountered within this study is around $9 \times 10^{11} \mathrm{~m}^{-3}$, resulting in $\mu$ falling to 0.975 and the differential absorption given by the integrand of (3) being around $2.5 \%$ too low. However, plotting time series of the radar data reveals that spikes of the same magnitude are common. These often result in nonsensical negative data, and are difficult to remove without skewing integrated values, for example, by excluding all negative valued data. In view of the considerable random errors in the radar data, we require the relationship between electron density and absorption to be linear and therefore choose to keep the approximation $\mu \approx 1$.

[16] The error introduced by using an effective collision frequency is greatest at low altitudes and falls very quickly with increasing height. With a receiver frequency of $38.2 \mathrm{MHz}$ for IRIS, the absorption is typically calculated to be less than $2 \%$ too large at $60 \mathrm{~km}$, and $0.1 \%$ too large at $70 \mathrm{~km}$. However, at $10 \mathrm{MHz}$ these values increase to $26 \%$ and $2 \%$, respectively, and at $5 \mathrm{MHz}$ they are $210 \%$ and $10 \%$.

[17] It is convenient to take the simple formulas calculated by Schunk and Nagy [1978] for the effective collision frequencies of the most populous atmospheric species. These are based on the cross sections given by Banks [1966], with the exception of atomic oxygen.

[18] The momentum-transfer cross section of atomic oxygen is difficult to determine experimentally; Schunk and Nagy [1978] took results from Henry and McElroy [1968], but we choose slightly more recent values by Thomas and Nesbet [1975]. The latter are a little higher over the temperature range of interest and closer to the values of Banks [1966]. The relative concentration of atomic oxygen increases with altitude; at $110 \mathrm{~km}$ it is around $12 \%$ of the total neutral density. Here the higher cross section values provided by Thomas and Nesbet [1975], and used in this study, translate to a rise of just $3 \%$ in the differential absorption.

[19] For molecular nitrogen we use momentum-transfer cross sections from Morrison et al. [1997]. Although these differ considerably from Banks [1966], especially at low energies, the variations largely cancel when integrated over the electron energy distribution and amount to less than $2.5 \%$ in the temperature range $150-500 \mathrm{~K}$. The updated effective momentum-transfer collision frequencies for use between $150 \mathrm{~K}$ and $500 \mathrm{~K}$, and employed in this study are:

$$
\left\langle\nu_{e, \mathrm{~N}_{2}}\right\rangle=N\left(\mathrm{~N}_{2}\right)\left(4.02+2.37\left(1-1.54 \times 10^{-4} \mathrm{~T}_{\mathrm{e}}\right) \mathrm{T}_{\mathrm{e}}\right) \times 10^{-17}
$$

$$
\left\langle\nu_{e, \mathrm{O}}\right\rangle=1.37 \times 10^{-16} N(\mathrm{O})\left(1+3.32 \times 10^{-4} \mathrm{~T}_{\mathrm{e}}\right) \mathrm{T}_{\mathrm{e}}^{\frac{1}{2}},
$$

where $N\left(\mathrm{~N}_{2}\right)$ and $N(\mathrm{O})$ are the densities of $\mathrm{N}_{2}$ and $\mathrm{O}$ respectively, in $\mathrm{m}^{-3}$. $T_{e}$ is the electron temperature in Kelvin.

[20] Cross sections for the other neutral species are taken directly from Banks [1966], noting that those for the other important species, $\mathrm{O}_{2}$, agree with more recent measurements by Sullivan et al. [1995], within the errors of their work.

\section{Method}

[21] A linear problem in inverse theory generally takes the form:

$$
c_{i}=\int r_{i}(x) u_{0}(x) d x+n_{i}
$$

where each $c_{i}$ is a measurement that contains information about a particular aspect of an unknown process, $u_{0}(x)$, and $n_{i}$ is the error of the $i$ th measurement. The measurements are related to the unknown process by the response kernel, $r_{i}(x)$. Given the measurements, their errors, and the response kernel, a statistical approximation, $\hat{u}_{0}(x)$, to the unknown process, $u_{0}(x)$, is sought. In our case $c_{i}$ is the height integrated absorption measured by IRIS and $r_{i}(x)$ are modeled absorption profiles, (given by (3)). These modeled profiles are calculated with electron densities measured by the EISCAT VHF radar together with the model neutral atmosphere, NRLMSISe-00. $u_{0}(x)$ is therefore a scaling factor for the modeled absorption, which, after a small iterative correction, gives a scaling factor for the modeled effective momentum-transfer collision frequency at each altitude. For this reason we denote the absorption scaling factor as $u_{0}(x)$, and the collision frequency scaling factor as $u(x)$. The radar range gates are discrete and the integral in (6) is accordingly replaced by a sum.

[22] By choosing our solution to be a scaling factor for model values the regularization is greatly simplified and, by re-running the neutral atmosphere model for each profile, diurnal variations of the neutral atmosphere are included a priori.

[23] The Backus-Gilbert method of regularization is utilized and briefly described below. For a more in-depth explanation see Press et al. [1996].

[24] We seek a solution to the inversion problem expressed in (6), that is a compromise between an exact solution to the data set and one that is reasonable. This is achieved by minimizing the expression:

$$
A+\lambda B
$$

where $A$ is a measure of the spread (the reciprocal of resolution) of the solution, i.e., how far the solution is away from an exact solution for the data set. $B$ is a measure of its instability, i.e., how unrealistic the solution is. $\lambda$ allows tuning of the result between a stable solution and one that best fits the specific data set.

[25] The resolution function, $\hat{\delta}\left(x, x^{\prime}\right)$, is implicitly defined by:

$$
\hat{u}_{0}(x)=\int \hat{\delta}\left(x, x^{\prime}\right) u_{0}\left(x^{\prime}\right) d x^{\prime}
$$




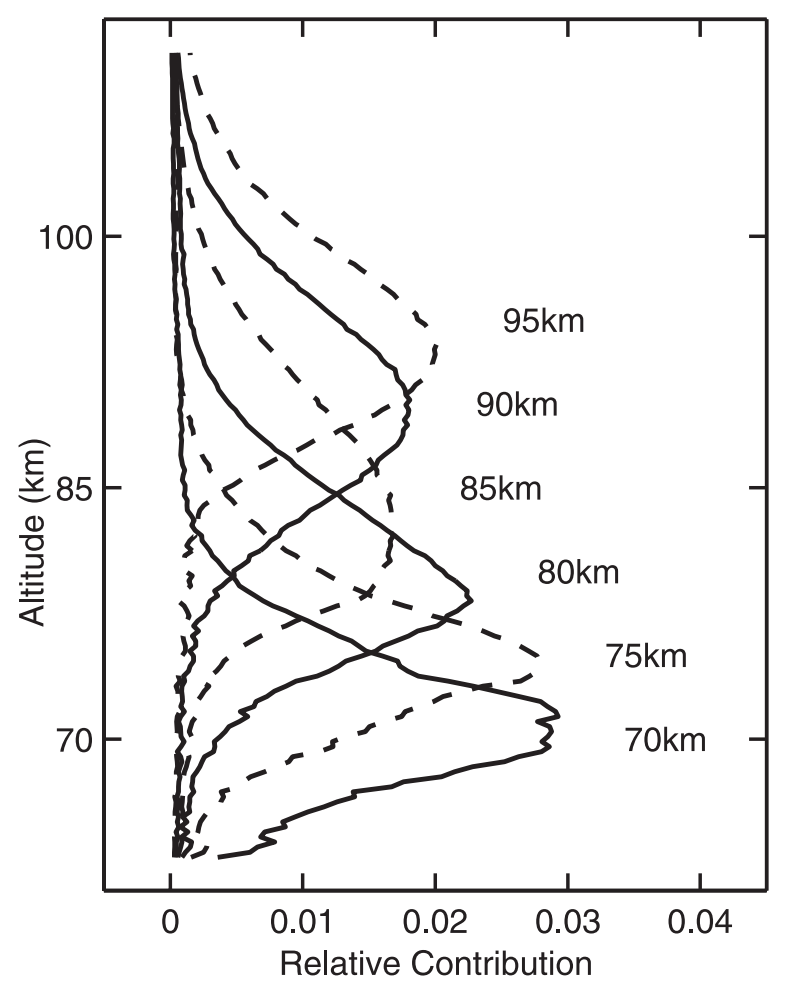

Figure 2. $\hat{\delta}$, the resolution function.

where $\hat{u}_{0}(x)$ is our smooth, statistical solution and $u_{0}\left(x^{\prime}\right)$ is the exact (though highly unstable) solution for the particular data set used. $x$ represents the altitude for which we are finding the solution, and $x^{\prime}$ the altitudes of the radar range gates. Minimizing $A$, to increase the resolution of the solution, is equivalent to bringing the resolution function closer to the identity matrix.

[26] Figure 2 shows the resolution function corresponding to our result, for several values of $x$. The value of $\lambda$ is reduced with altitude in order to keep a reasonable resolution across the entire range of $x$.

[27] An error value, $n_{i}$, is assigned to each absorption measurement, $c_{i}$. These control the weight given to each sample, as well as defining the error bounds of the final result through the standard propagation of errors. The Backus-Gilbert method provides a solution for $\hat{u}_{0}(x)$, together with the corresponding errors.

[28] It is useful to define the set of inverse response kernels, $q_{i}$ :

$$
\hat{u}_{0}(x)=\sum_{i} q_{i}(x) \cdot c_{i}
$$

and the integrated response kernel, $R_{i}$ :

$$
R_{i}=\int r_{i}(x) d x
$$

$B$, the measure of the stability of the solution, is given by:

$$
B \equiv \operatorname{var}\left[\hat{u}_{0}(x)\right]=\mathbf{q}(\mathbf{x})^{\mathbf{T}} \mathbf{S} \mathbf{q}(\mathbf{x}),
$$

where $S_{i j}$ is the covariance matrix. The errors, $n_{i}$, are herein assumed to be independent, therefore:

$$
S_{i j}= \begin{cases}n_{i}^{2}, & \text { if } \mathrm{i}=\mathrm{j}, \\ 0 & \text { if } \mathrm{i} \neq \mathrm{j} .\end{cases}
$$

$A$, the measure of the width or spread of $\hat{\delta}\left(x, x^{\prime}\right)$, is given by:

$$
A=\mathbf{q}(\mathbf{x})^{\mathbf{T}} \mathbf{W}(\mathbf{x}) \mathbf{q}(\mathbf{x})
$$

where $W_{i j}(x)$ is the spread matrix:

$$
W_{i j}(x) \equiv \int\left(x^{\prime}-x\right)^{2} r_{i}\left(x^{\prime}\right) r_{j}\left(x^{\prime}\right) d x^{\prime} .
$$

This definition, used by Backus and Gilbert in their method, is one of many possible definitions.

[29] Equation (7) can now be written in terms of $S_{i j}$ and $W_{i j}(x)$ as:

$$
A+\lambda B=\mathbf{q}(\mathbf{x})^{\mathbf{T}}[\mathbf{W}(\mathbf{x})+\lambda \mathbf{S}] \mathbf{q}(\mathbf{x}) .
$$

[30] The solution to the set of inverse response kernels is:

$$
\mathbf{q}(\mathbf{x})=\frac{[\mathbf{W}(\mathbf{x})+\lambda \mathbf{S}]^{-1} \mathbf{R}}{\mathbf{R}^{\mathbf{T}}[\mathbf{W}(\mathbf{x})+\lambda \mathbf{S}]^{-1} \mathbf{R}}
$$

where $\mathbf{R}$ is the integrated response kernel.

[31] The result of multiplying the inverse response kernels, $q_{i}(x)$, by the measurements, $c_{i}$, can be used to indicate whether a particular part of the data set is responsible for contributing a greater or lesser amount than expected toward the measurement values. This is particularly useful in determining whether a specific feature in the data set, in our case the electron density data, is real, or an instrumental effect.

[32] Finally, combining (9) and (11), we have:

$$
\hat{u}_{0}(x)=\mathbf{c}^{\mathbf{T}} \mathbf{q}(\mathbf{x}) \pm \sqrt{\mathbf{q}(\mathbf{x})^{\mathbf{T}} \mathbf{S} \mathbf{q}(\mathbf{x}) .}
$$

[33] This solution is computationally demanding, requiring the inversion of an $N \times N$ matrix for each altitude value, $x$, where $N$ is the number of measurements, $c_{i}$.

[34] Once the differential absorption scaling factor, $\hat{u}_{0}(x)$, has been calculated, the collision frequency scaling factor can be found with an iterative formula based on the integrand of (3).

$$
\begin{gathered}
\hat{u}_{n+1}(x)=\hat{u}_{0}(x)\left(\frac{\hat{u}_{n}(x)^{2}+\frac{\left(\omega \pm \omega_{H} \cos \theta\right)^{2}}{\nu_{\mathrm{mm}}(x)^{2}}}{1+\frac{\left(\omega \pm \omega_{H} \cos \theta\right)^{2}}{\nu_{\mathrm{mm}}(x)^{2}}}\right), \\
\hat{u}(x) \equiv \hat{u}_{n \rightarrow \infty}(x)=\frac{\nu_{\mathrm{um}}(x)}{\nu_{\mathrm{mm}}(x)},
\end{gathered}
$$

where $\nu_{\mathrm{mm}}$ is the modeled effective momentum transfer collision frequency, $\nu_{\mathrm{um}}$ is the unknown effective momen- 


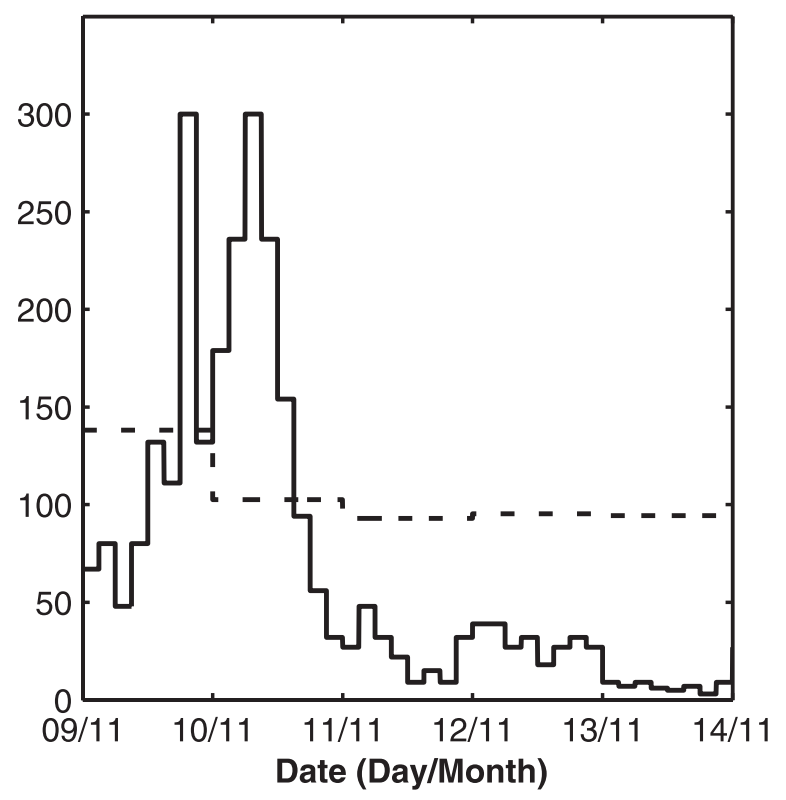

Figure 3. 3-h values of ap index (solid line) with daily $F_{10.7}$ index (dashed line).

tum transfer collision frequency, and $\omega$ is the angular wave frequency. This step is necessary when studying absorption from a lower altitude or using a riometer with a lower receiving frequency, since $\omega^{2} \gg \nu_{m}^{2}$ may no longer hold. In such cases one would also have to take care when calculating the modeled absorption, as discussed in section 3 .

[35] Notes within the Fortran code of the NRLMSISe-00 neutral atmosphere model [Picone et al., 2002], state that " $F_{10.7}, F_{10.7} a$ (an 81-day running mean of $F_{10.7}$ ), and ap effects are neither large nor well established below $80 \mathrm{~km}$ and these parameters should be set to 150, 150, and 4 respectively." For the NRLMSISe-00 model input we use these suggested values at all altitudes $(\approx 60-110 \mathrm{~km})$ and throughout the event. This simplifies interpretation of the results when splitting the data between periods of active and quiet conditions, and avoids a discontinuity at $80 \mathrm{~km}$.

\section{Data}

[36] Between 09 November 2004 to 13 November 2004 a unique set of data was recorded by EISCAT and IRIS. Over the 5 day period geomagnetic conditions changed from very active to quiet, there was little in the way of solar radio emissions to contaminate IRIS measurements, and polar cap absorption (PCA) [Reid, 1974] provided "illumination" of the lower D-region.

[37] Figure 3 shows that active conditions (ap $>50)$ lasted until 10 November 2004 21:00 UT and were followed by a quiet period, during which the value of ap remained below 50 for the rest of the data set.

[38] In Figure 4 the height-integrated absorption measured by beam 9 of IRIS is shown, together with a color plot of the modeled absorption based on radar data, which makes up the response kernel. The EISCAT VHF radar's power profile data with $1 \mathrm{~min}$ integration and $300 \mathrm{~m}$ range gates is used. As expected, a clear correlation can be seen between patches of strong modeled absorption and high levels of measured absorption. Exceptions can be seen around midday, especially on 11 November 2004.

\subsection{Excluded Data}

[39] Figure 5 shows four subsets of data, each containing periods of poor correlation between the absorption modeled using EISCAT data and that measured by IRIS. These are excluded from the calculation.

[40] In Figure 5a the correlation is excellent until around 10 November 2004 05:15 UT, when the larger beam of IRIS has a smoothing effect on the now spatially non-uniform (as confirmed by IRIS images) ionosphere. Both peaks and troughs become less pronounced in the IRIS measurements.

[41] Polar mesospheric winter echoes (PMWE) [Ecklund and Balsley, 1981] contaminate the radar data and, subsequently, the modeled absorption in Figure 5b. This occurs around midday on most of the days in the data set, and is usually accompanied by a significant contribution to absorption in IRIS from PCA at altitudes below the range of the radar, rendering data from these periods useless. Hargreaves [2005] reports that altitudes between 45 and $65 \mathrm{~km}$ typically account for $80 \%$ of the total daytime absorption during PCA events. For 2-3 h toward the beginning and end of these periods, Figure 4 shows that the absorption tapers off within the altitude range of the radar.

[42] Beginning 12 November 2004 14:40 UT and lasting a few hours, a small localized patch of absorption overlaps with the eastern extent of beam 9, and is seen more strongly in the neighboring beam to the east. It appears stationary within the IRIS images and is not seen in the radar (Figure 5c). The modeled absorption matches well with the neighboring IRIS beam to the west. Toward the end of this period large, shifting patches of absorption are seen advancing northward in the IRIS images. They enter IRIS beam 9 around a minute earlier than the radar (Figure $5 \mathrm{~d}$ ).

[43] With the exception of Figure $5 \mathrm{~b}$ these problems should be eliminated by using a riometer and radar with comparable fields of view.

[44] Additionally the period 10 November 2004 18:10 UT to 11 November 2004 01:00 UT is also excluded. This covers three strong patches of enhanced electron density that seem to have little to no effect on the absorption measured by IRIS. When included, this period affects the result significantly; it is thought that the patches are very localized (i.e., smaller than the riometer beam).

\subsection{Sources of Error}

[45] As well as the expected instrument errors encountered in the measurements, prominent sources of error include: calibration of the radar data, errors in the riometer quiet day curve and the different fields of view of the riometer and radar beams (see Figure 1).

[46] The field of view of the 9th beam of IRIS is closest to that of the vertically pointing radar, intersecting it at $\approx 80 \mathrm{~km}$ (Figure 1). Although it is possible to interpolate between the riometer beams for the location of the radar beam at each altitude, the correlation between the instruments is hampered by the contribution to the other riometer beams from absorption further away from EISCAT. Fortunately, the absorption profiles are centered at approximately $80 \mathrm{~km}$, making the pointing direction of beam 9 ideal. 
(gp) әџе6 әбиел ıерел ıәd uo!!dıosq

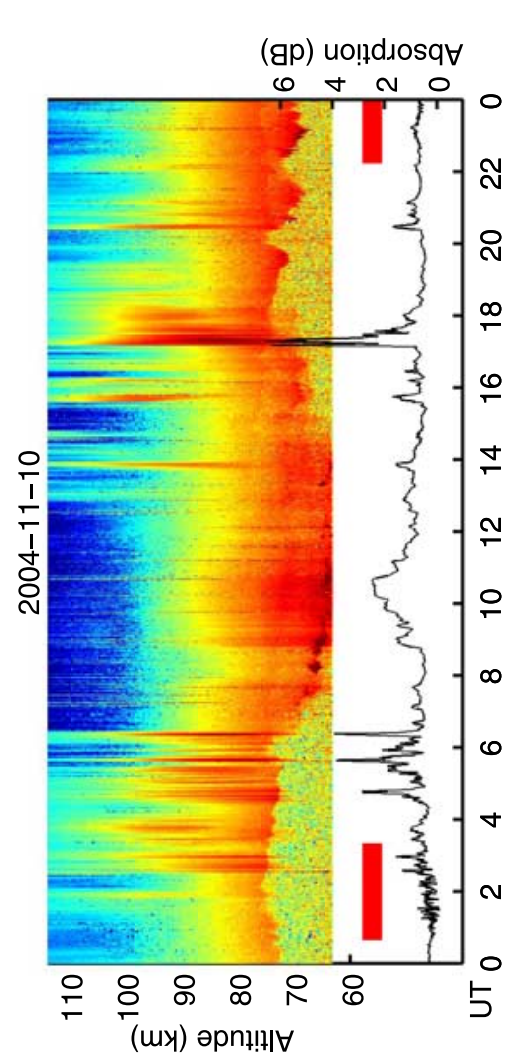

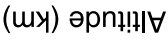

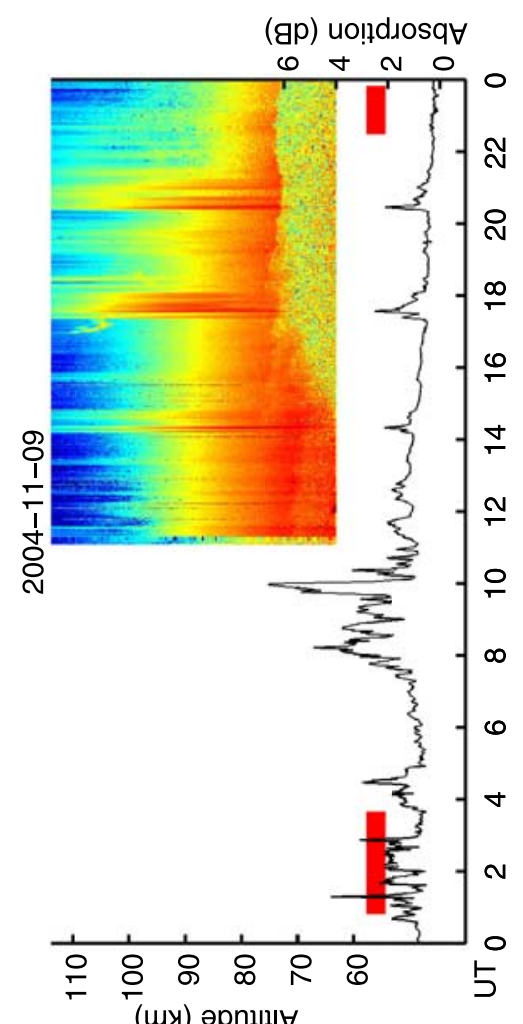

(uy) әрn+!!l|

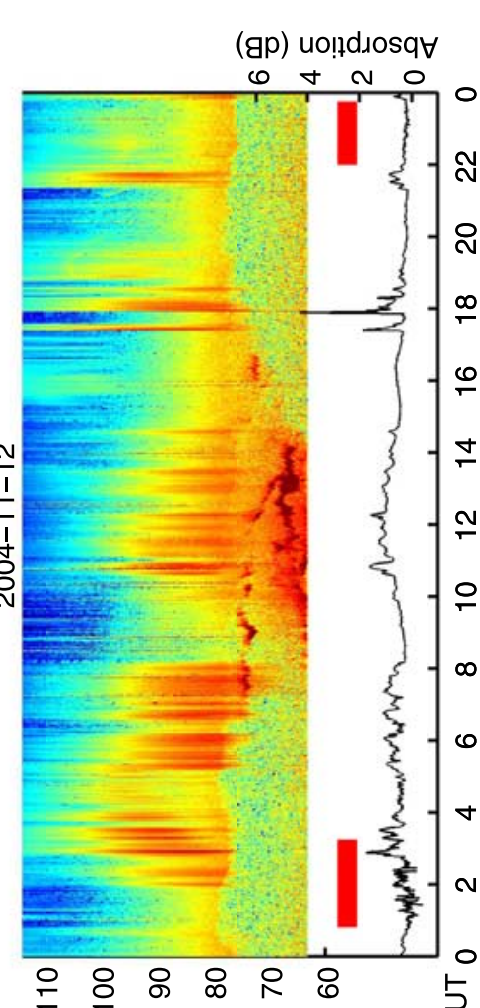

(uY) әрпџ!!I

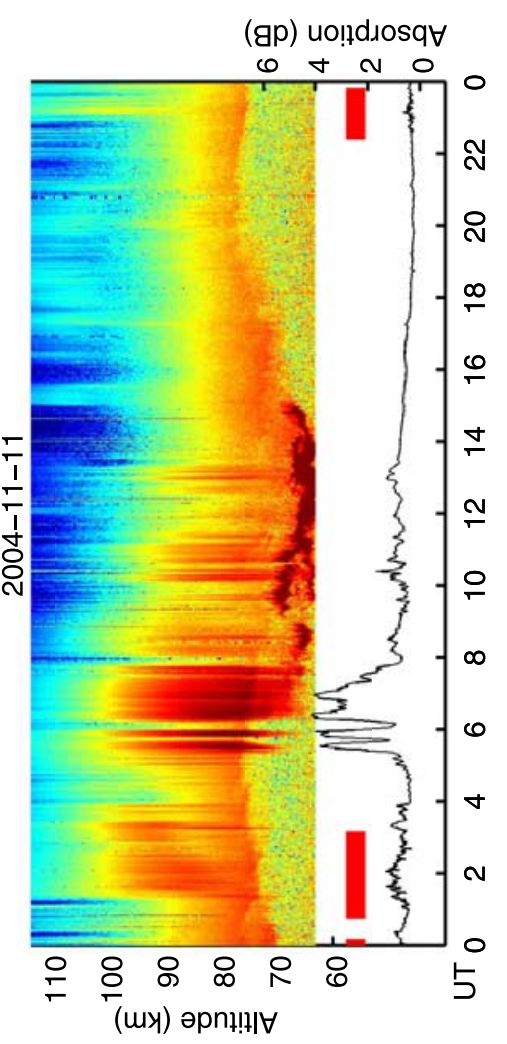

$\bar{c}$
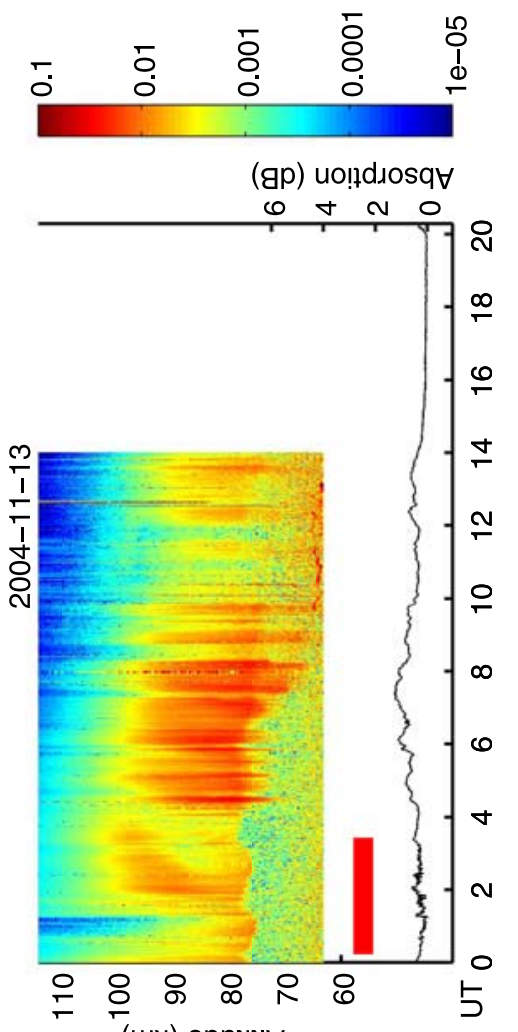

(uy) әpn!!+ly

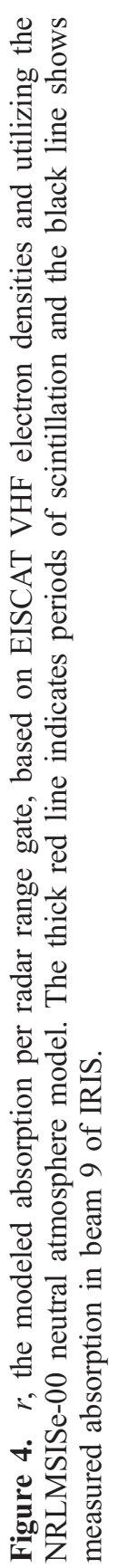



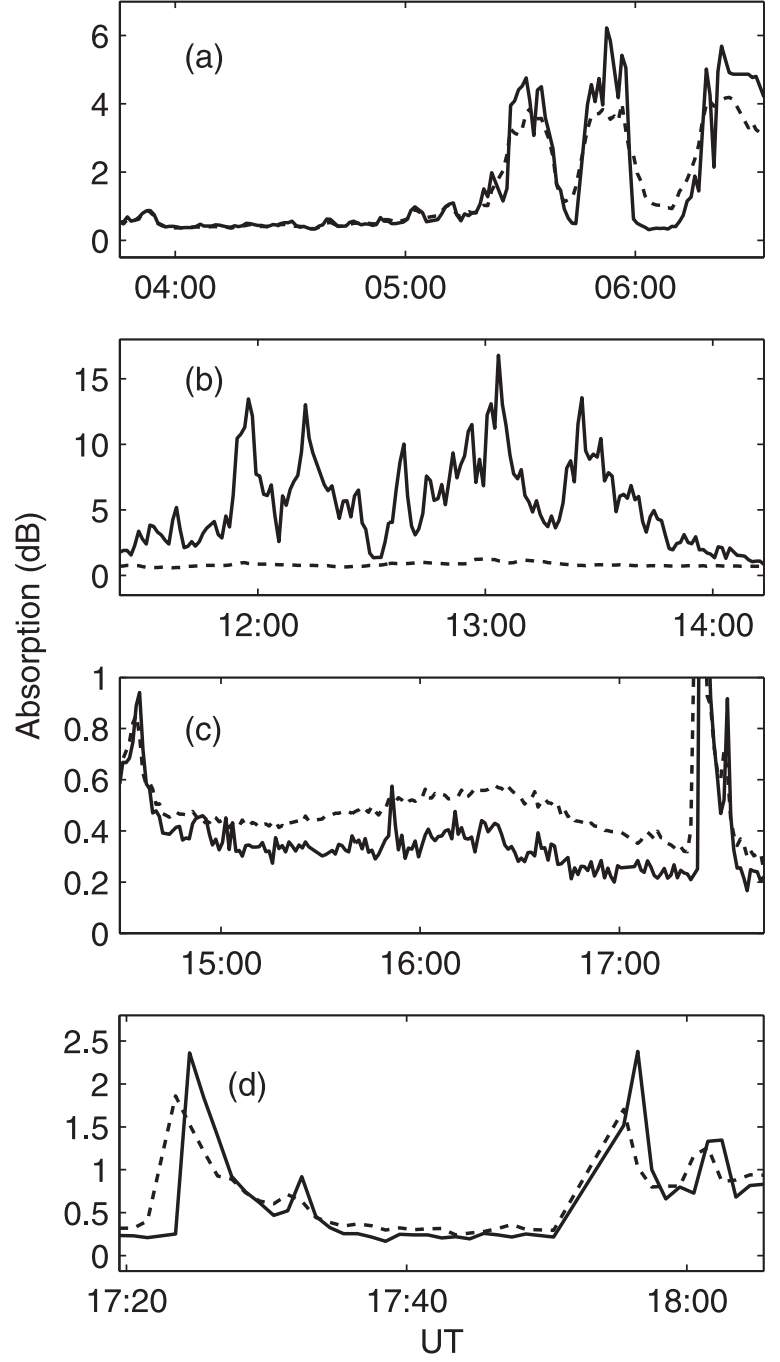

Figure 5. Subsets of data that show periods of poor correlation between absorption calculated using EISCAT and NRLMSIS (solid line) and measured by beam 9 of IRIS (dashed line). (a), (b) are 11 November 2004; (c), (d) are 12 November 2004. For further explanation see text.

[47] There are difficulties in calibrating the EISCAT VHF data directly with the dynasonde. Instead it is calibrated against electron density measurements from the EISCAT UHF radar, which is itself calibrated against the dynasonde. Comparing many samples of the UHF and VHF data from an overlapping altitude range on 13 November 2004, the median electron density ratio is found to be 1.84 , the mean 1.90 and the total error in the mean $0.144(\approx 8 \%)$. The median value being the better choice as the values are not symmetrically distributed about the mean.

[48] There are no error values given with the EISCAT power-profile data but an estimate of the random error can be taken from the data by comparing consecutive samples. The estimated error of the integrated modeled absorption, $R_{i}$, carried through from the radar data is:

$$
\sigma_{R_{i}} \approx \sqrt{\sum_{n} \frac{\left(r_{i, n}-r_{i, n+1}\right)^{2}}{2}} \approx 0.025 \mathrm{~dB} .
$$

[49] Let us define $m_{i}$ as the measured absorption, $c_{i}$, minus the modeled absorption, $R_{i}$ :

$$
m_{i}=c_{i}-R_{i} .
$$

$\sigma_{R_{i}}$ is close to the noise-limit of IRIS and is small compared to the width of the distribution of $m_{i}$ for this data set, which is symmetrical but has a longer-than-Gaussian tail. This larger variability between the modeled (radar) and measured (riometer) values is almost certainly due to the separation of the instruments. Although it is impossible to know how the colocation error is distributed in altitude, assuming that it is constant allows for the use of combined error values, $n_{i}$. It is logical to assume, and can be clearly seen in the data, that this colocation error increases with increasing absorption.

[50] Plotting the standard deviation of $m_{i}$ for several subsets of data, binned according to $c_{i}$, gives an idea of the relationship between absorption, $c_{i}$, and combined (colocation and instrument) error. A good approximation, which forms the basis of our error values, is found to be:

$$
n_{i}= \pm \sqrt{\left([0.5 \mathrm{~dB}]^{2}+\left[0.5 \times \mathrm{c}_{\mathrm{i}}\right]^{2}\right)} .
$$

[51] Several hours of scintillation occurred each night between approximately 22:00 UT and 03:30 UT (red lines, Figure 4). The error estimate for these periods is increased accordingly.

[52] It should be noted that multiplying every value of $n_{i}$ by a constant has no effect on the final result, but the errors of the result scale proportionally with the constant.

\section{Results}

[53] Figure 6 shows that the effective momentum-transfer collision frequency profiles calculated using the NRLMSISe00 model are very accurate in the $68-95 \mathrm{~km}$ range, with correction factors that are close to unity during both the active and quiet periods. Above $95 \mathrm{~km}$ the larger error indicates that the stability of the result is poor, this is due to the small contribution to the total absorption and a uniformity of absorption with respect to height at these altitudes. The apparent rise in the correction factor above $95 \mathrm{~km}$, in both the active and quiet periods, is therefore thought to be a coincidence rather than a real trend. Generally, an absorption profile with an unvarying shape will present a badly posed inversion problem, supplying little information of the real-life contribution to integrated absorption (and thus collision frequency) from each altitude.

[54] Below $68 \mathrm{~km}$ the result is smaller than unity, and less certain than might be expected. Clutter in the arc_dlayer-v program on the EISCAT VHF system was significantly reduced in June 2004 but may still remain a factor, (I. Häggström, private communication, 2007). This could be responsible for systematic overestimating in the power profile data from the lower range gates, leading to a reduced correction factor. The added uncertainty is caused by larger errors inherent in measuring very small electron densities, together with the rarity of patches of significant absorption at low altitudes.

[55] The result for the entire data set, Figure 6a, is closer to that of the quiet period, Figure $6 \mathrm{c}$, simply because the 

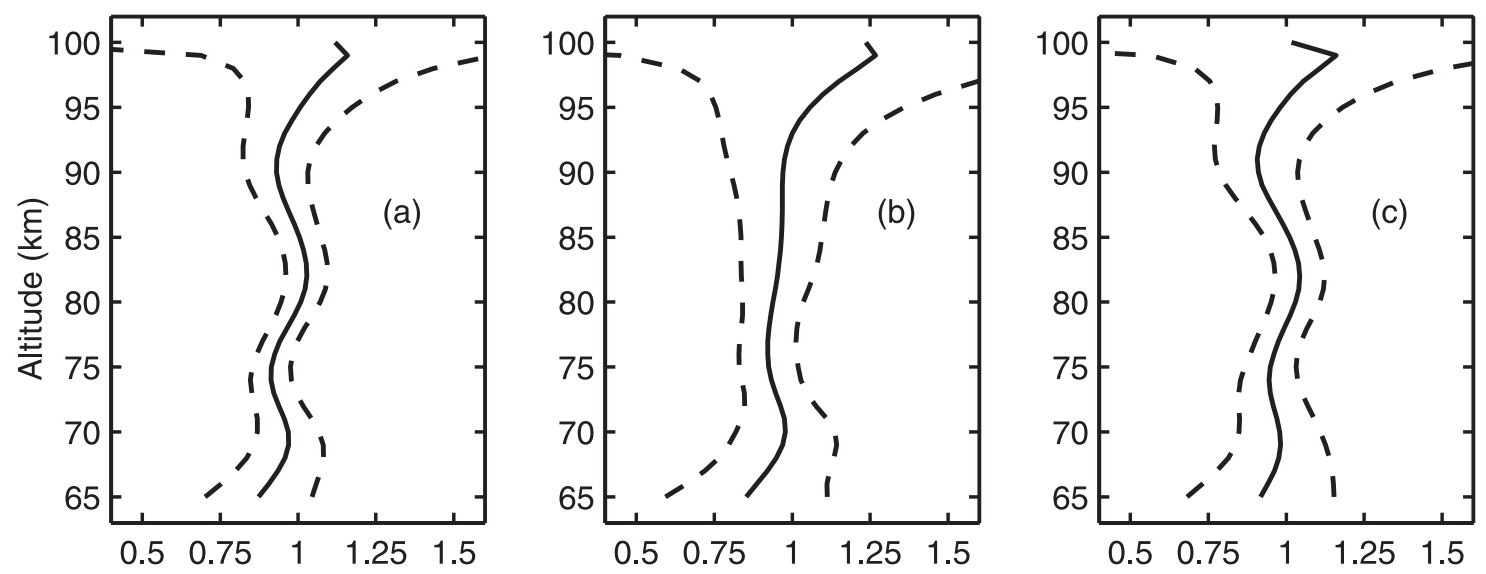

Figure 6. Correction factor, $\hat{u}$, for the effective momentum-transfer collision frequency given by NRLMSISe-00 (solid lines), with error bounds (dashed lines). (a) All of the data; (b) Active conditions $($ ap $>50)$; (c) Quiet conditions $($ ap $<50)$.

majority of the data falls within the quiet period. The mean values of each correction factor profile between $70 \mathrm{~km}$ and $93 \mathrm{~km}$, where the result is reasonably stable, are 0.965 , 0.956 and 0.974 , for all of the data, the active period and the quiet period respectively. Each of these factors differ from unity within the error of the calibration of the radar data $(\approx 8 \%)$. Therefore the effect of the ap index on the effective collision frequency in the D-region is minimal, and less than the errors of our result. This confirms the assumption that "ap effects are neither large nor well established below $80 \mathrm{~km}$ ", suggested in the NRLMSISe00 source code.

[56] Without any regularization the inversion would be very badly posed, and the resultant profiles would oscillate wildly. In making the result smooth, the regularization has an effect similar to that of a low-pass filter. Some residual oscillation is present in each of the profiles, but it is important that we do not lose real features by overly constraining the result.

[57] Multiplying the effective collision frequency profiles, calculated with values from the neutral atmosphere model NRLMSISe-00, by the correction profiles (Figure 6), gives corrected effective collision frequency profiles. The corrected profiles for 10 November 2004 00:00 UT (active conditions) and 12 November 2004 00:00 UT (quiet conditions), are shown in Figure 7.

[58] In order to check that our regularization is not confining the result (beyond a reasonable amount) to the a priori information, we run the program with various changes to the response kernel, $r$. Each profile of $r$ (the model absorption per radar range gate) is multiplied by the functions depicted on the left hand side of Figure 8 and the resulting variation in the solution, $\hat{u}_{0}$, is shown in the right hand side. For example, the top plot shows the effect of increasing the NRLMSISe-00 calculated absorption by $20 \%$ at all altitudes. Ideally, the solution would then be compensated by a reduction of $100 \times(1-1 / 1.2)=16.67 \%$ at all altitudes.

[59] The changes in our result match very well with the ideal response for slowly varying functions of altitude such as the first two examples, but narrow layers are less resolved. This is a reflection of the sensitivity of the result to similar variations in the actual neutral atmosphere.
[60] Inspection of the set of inverse response kernels reveals that, when included in the data set, PMWE contributes far less to the measured absorption than would be expected from such an increase in electron density. The same is true for the apparent enhancement in the EISCAT data (Figure 4) at an altitude of $100-105 \mathrm{~km}$ on 09 November 2004 17:00 UT. Neither are thought to be real enhancements of electron density.

\section{Conclusion}

[61] We have demonstrated a new technique for measuring the effective electron-neutral momentum-transfer colli-

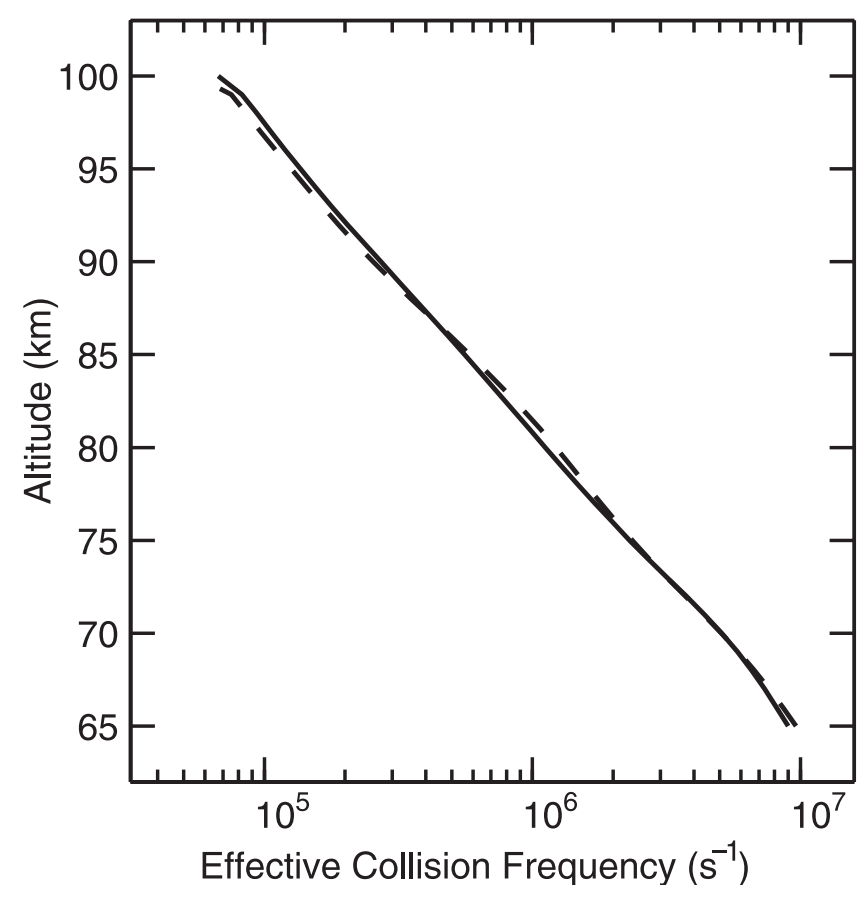

Figure 7. Corrected effective collision frequency profiles during active conditions at 10 November 2004 00:00 UT (solid line) and quiet conditions at 12 November 2004 00:00 UT (dashed line). 


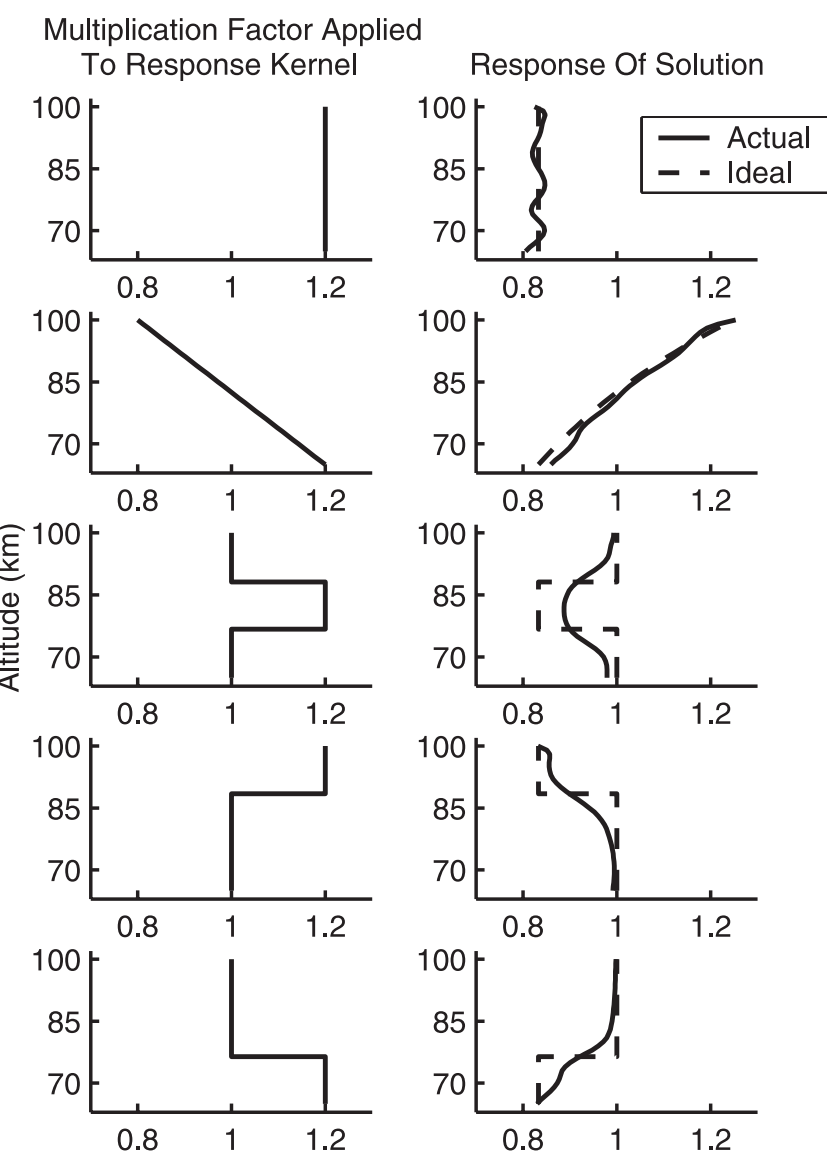

Figure 8. Response of the solution $\hat{u}_{0}(x)$ (right), to the multiplication factor profiles (left) imposed on the response kernel, $r_{i}(x)$.

sion frequency profile in the D-region. The results match well with values derived from the NRLMSISe-00 neutral atmosphere model and show no detectable variation between the active and quiet periods. The method would be improved by using a riometer located at the radar site, with a comparable field of view. This would allow for higher altitude resolution, while reducing the errors (residual oscillations) in the result. Figure 1 suggests that ARIES should provide a significant improvement in this respect, when processed data becomes available.

[62] Direct comparison of the results with previous works is complicated by multiple definitions of the electronneutral collision frequency. In the case of $\nu_{M}$ defined by Sen and Wyller's magneto-ionic theory, an added inconsistency arises from the invalid assumptions of temperature independent collisional cross-sections.

[63] The corrected collision frequency profiles (Figure 7) are in close agreement with the results of Miyazaki [1975] from a geographic latitude of $69^{\circ} 00^{\prime} \mathrm{S}$. This is close to the southern hemisphere latitude corresponding to Tromsø $\left(69^{\circ} 35^{\prime} \mathrm{N}\right)$, and confirms that the log linear altitude dependence assumed by Miyazaki [1975] is a reasonable approximation in the high-latitude D-region.

[64] Acknowledgments. With thanks to A. Senior for his invaluable input and S. Marple for providing the mapping and beam plotting software used in generating Figure 1. The Imaging Riometer for Ionospheric Studies (IRIS) is funded by the Science and Technology Facilities Council (STFC) and operated by the Department of Communications Systems at Lancaster University (UK) in collaboration with the Sodankylä Geophysical Observatory. EISCAT is an international association supported by the research councils of Finland (SA), France (CNRS), Germany (MPG), Japan (NIPR), Norway (NFR), Sweden (VR) and the United Kingdom (STFC)

[65] Amitava Bhattacharjee thanks the reviewers for their assistance in evaluating this paper.

\section{References}

Banks, P. (1966), Collision frequencies and energy transfer, Planet. Space Sci., 14, 1085-1103.

Baron, M. (1986), EISCAT progress 1983-1985, J. Atmos. Terr. Phys., 48 , $767-772$.

Browne, S., et al. (1995), An imaging riometer for ionospheric studies, J. Electron. Commun. Eng. Jpn., 7, 209-217.

Chapman, S., and T. G. Cowling (1970), The Mathematical Theory of NonUniform Gases, Cambridge Univ. Press, Cambridge, U.K.

Davies, K. (1966), Ionospheric Radio Propagation, Dover Publications Inc., New York.

Drob, D. P., and J. M. Picone (2000), Statistical performance measures of the HWM-93 and MSISe-90 empirical atmospheric models and the relation to infrasonic CTBT monitoring, 22nd Annual Seismic Research Symposium, New Orleans, Louisiana, USA.

Ecklund, W. L., and B. B. Balsley (1981), Long-term observations of the Arctic mesosphere with the MST radar at Poker Flat, Alaska, J. Geophys. Res., 86(A9), 7775-7780.

Flemming, E. S., et al. (1988), Monthly mean global climatology of temperature, wind, geopotential height and pressure for $0-120 \mathrm{~km}, N A S A$ Technical Memorandum, 100697.

Garrett, A. J. M. (1985), Multi-species kinetic generalization of the AppletonHartree dispersion formula, J. Plasma Phys., 33(2), 265-284.

Gokov, A. M., et al. (1991), Simultaneous measurement of the density and frequency of electron collisions with molecules in the ionospheric Dregion, Radiophys. Quant. Electron., 34(3), 275-277.

Grill, M., et al. (2003), A New Imaging Riometer based on Mills Cross Technique, paper presented at 7th Int. Symp. on Communication Theory and Applications, July 2003, Ambleside, UK, pp. 26-31.

Hargreaves, J. K. (2005), A new method of studying the relation between ionization rates and radio-wave absorption in polar-cap absorption events, Ann. Geophys., 23(2), 359-369.

Hedin, A. E. (1991), Extension of the MSIS thermosphere model into the middle and lower atmospheres, J. Geophys. Res., 96(A2), 1159-1172.

Henry, R. J. W., and M. B. McElroy (1968), The Atmospheres of Venus and Mars, Gordon and Breach, New York.

Itikawa, Y. (1971), Effective collision frequency of electrons in atmospheric gases, Planet. Space Sci., 19, 993-1007.

Miyazaki, S. (1975), Relation between lower ionospheric electron density profiles and cosmic noise absorption during auroral zone disturbances, J. Geomagn. Geoelectr., 27, 113-129.

Morrison, M., et al. (1997), Ultrasimple calculation of very low energy momentum-transfer and rotational-excitation cross sections: $e-\mathrm{N}_{2}$ scattering, Phys. Rev. A, 55(4), 2786-2798.

Picone, J. M., et al. (2002), NRLMSISE-00 empirical model of the atmosphere: Statistical comparisons and scientific issues, J. Geophys. Res., 107(A12), 1468, doi:10.1029/2002JA009430.

Press, W. H., et al. (1996), Numerical Recipes in Fortran 77, 2nd ed., Cambridge Univ. Press, Cambridge, U.K.

Reid, G. C. (1974), Polar-cap absorption - Observations and theory, Fundam. Cosmic Phys., 1, 167-202.

Schunk, R. W., and A. F. Nagy (1978), Electron temperatures in the F region of the ionosphere: Theory and observations, Rev. Geophys. Space Phys., 16(3), 355-399.

Sen, H. K., and A. A. Wyller (1960), On the generalization of the AppletonHartree magnetoionic formulas, J. Geophys. Res., 65(12), 3931-3950.

Serafimova, M., and K. Serafimov (1991), On a possibility for continuous ground-based measurements of the collision frequency in the ionosphere, B'lgarska Akad. Nauk. Dokl., 4410, 35-38.

Sullivan, J. P., et al. (1995), Low energy electron scattering from $\mathrm{O}_{2}$, J. Phys B At. Mol. Opt. Phys., 28, 4319-4328.

Thomas, L. D., and R. K. Nesbet (1975), Low energy electron scattering by atomic oxygen, Phys. Rev. A, 12(4), 1729-1730.

Vuthaluru, R. A., et al. (2002), Collision frequencies in the D-region, J. Atmos. Sol. Terr. Phys., 64, 2043-2054.

M. Beharrell and F. Honary, Department of Communication Systems, InfoLab21, University of Lancaster, Lancaster, LA1 4WA, United Kingdom. (m.beharrell@lancaster.ac.uk) 